\title{
Combined clerking - streamlining emergency admissions
}

\author{
Authors: Holly Gilbert, Dominic Reynish, Dan Richter, Ian Neville, Kamy Thavanesan, Mark Sopher, David Martin, \\ Robert Willington, Richard Byrom, Imran Ghafoor and Alyson O’Donnell
}

\section{Introduction}

Conventional clerking by the emergency department (ED) followed by specialty clerking involves duplication of information. Having multiple documentation lengthens clerking time, slows patient flow and takes longer to review during the admission. The Royal Bournemouth Hospital is a district general hospital on the south coast. The trust sought to improve the efficiency by reducing duplication as part of a quality improvement (QI) team focusing on the first 24 hours of the patient journey.

\section{Methods}

Our group was formed by open invitation. The QI team organised weekly open meetings and included consultant representatives from the ED, surgery, acute medicine, elderly care, stroke and cardiology. It also consisted of a chief registrar, core medical trainee, QI manager, nurses, pharmacists, physiotherapists and occupational therapists. By agreeing and analysing a process map for our admission process, we highlighted areas of potential improvement, and then focused our priorities using an Ease-Impact (PICK) matrix.

Our aim was to contribute towards the trust's plan for winter pressures, and we organised our plan on a Gantt chart accordingly. It was agreed that we should aim to work collaboratively and that documentation is part of a continuum of care. We designed and implemented combined admission documentation for both ED and specialties to use collaboratively which starts on patients' arrival and continues into their admission. This was implemented on 29 October 2018.

We measured time from ED arrival to post-take ward round (PTWR) as a measure of efficiency, analysing sample data from our electronic medical take list covering two Wednesdays before and two Wednesdays after our intervention, with a 'buffer' of a week each side of the implementation to discount any short term unrepresentative effects. We corroborated this through a manual check of 40 random patient records. We also collected qualitative data throughout the process, using questionnaires to gather feedback for the multiple Plan, Do, Study, Act cycles to improve the process.

Authors: Royal Bournemouth Hospital

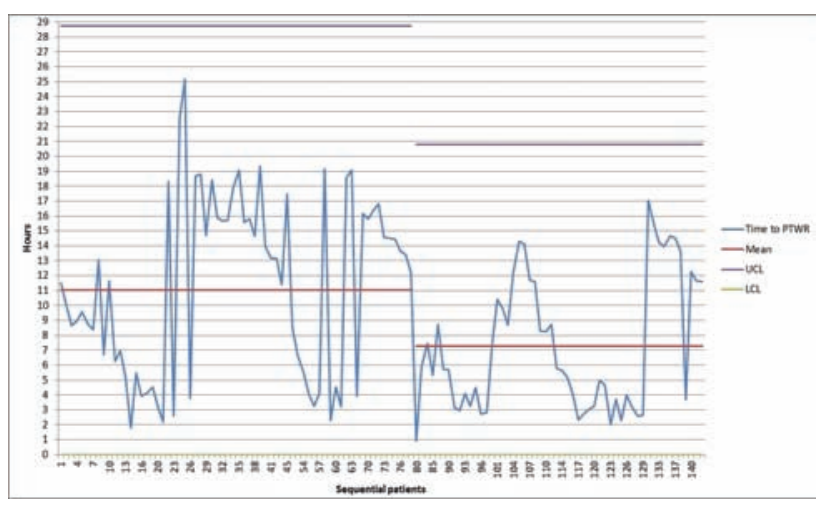

Fig 1. Time to PTWR on sample Wednesdays, pre- and post-combined clerking

\section{Results}

Our data showed that mean average ED arrival to medical review was faster by $2 \mathrm{hr} 32 \mathrm{~min}$ ( $5 \mathrm{hr} 10 \mathrm{~min}$ to $2 \mathrm{hr} 38 \mathrm{~min}$ ) and ED arrival to PTWR was faster by $3 \mathrm{hr} 45 \mathrm{~min}$ ( $11 \mathrm{hr} 2 \mathrm{~min}$ to $7 \mathrm{hr} 17 \mathrm{~min})$.

Questionnaire feedback from staff was largely positive and constructive during the process and alterations were made according to their suggestions.

\section{Conclusions}

This project improved visibility of ED documentation, reduced unnecessary duplication, reduced waiting times and overall improved efficiency for staff and patients. It was widely supported by ward staff and management, thus ensuring its sustainability.

This method is a middle ground between 'single' clerking and conventional (ie 'double') clerking. It reaps many of the benefits seen with single clerking but with minimal change to the organisational processes. Patient flow was faster and this combined process could be implemented in other trusts without significant difficulty.

Looking to the future, we hope to improve the system further with more automation of data collection such as digital admission documentation to further improve the admission process for patients and staff. 